\title{
CARDINAL CONDITIONS FOR STRONG FUBINI THEOREMS
}

\author{
JOSEPH SHIPMAN
}

\begin{abstract}
If $\kappa_{1}, \kappa_{2}, \ldots, \kappa_{n}$ are cardinals with $\kappa_{1}$ the cardinality of a nonmeasurable set, and for $i=2,3, \ldots, n \quad \kappa_{i}$ is the cardinality of a set of reals which is not the union of $\kappa_{i-1}$ measure-0 sets, then for any nonnegative function $f: \mathbf{R}^{n} \rightarrow \mathbf{R}$ all of the iterated integrals

$$
I_{\sigma}=\iint \cdots \int f\left(x_{1}, x_{2}, \ldots, x_{n}\right) d x_{\sigma(1)} d x_{\sigma(2)} \cdots d x_{\sigma(n)}, \quad \sigma \in S_{n},
$$

which exist are equal. If all $n !$ of the integrals exist, then the weaker condition of the case $n=2$ implies they are equal. These cardinal conditions are consistent with and independent of ZFC, and follow from the existence of a real-valued measure on the continuum. Other necessary conditions and sufficient conditions for the existence and equality of iterated integrals are also treated.
\end{abstract}

\section{INTRODUCTION}

In its most familiar form, Fubini's theorem asserts that if $f: \mathbf{R}^{2} \rightarrow \mathbf{R}$ is a measurable function, then the iterated integrals $\int\left[\int f(x, y) d x\right] d y$ and $\int\left[\int f(x, y) d y\right] d x$ exist and are equal. This paper will investigate the conditions under which the assumption " $f$ is a measurable function" can be weakened. Assertions about the existence and equality of iterated integrals of functions $f: \mathbf{R}^{n} \rightarrow \mathbf{R}$ which are not necessarily measurable will be called "strong Fubini theorems".

In the following example $f:[0,1)^{2} \rightarrow \mathbf{R}$ has disagreeing iterated integrals:

$$
f(x, y)= \begin{cases}2^{2 n}, & \text { if } 1-\frac{1}{2^{n}} \leq x, y<1-\frac{1}{2^{n+1}} \\ -2^{2 n+1}, & \text { if } 1-\frac{1}{2^{n}} \leq y<1-\frac{1}{2^{n+1}} \leq x<1-\frac{1}{2^{n+2}} \\ 0, & \text { otherwise. }\end{cases}
$$

As is easily computed, $\iint f d x d y=0$ but $\iint f d y d x=\frac{1}{4}$. The only "bad" point is $(1,1)$, in any neighborhood of which $f$ is unbounded in both directions ( $f$ can even be made continuous on $[0,1)^{2}$ by using "tent functions").

Received by the editors July 12, 1989.

1980 Mathematics Subject Classification (1985 Revision). Primary 03E15, 28A35; Secondary 03E35, 03E65, 28A20. sets.

Key words and phrases. Fubini theorem(s), real-valued measurable cardinals, nonmeasurable 


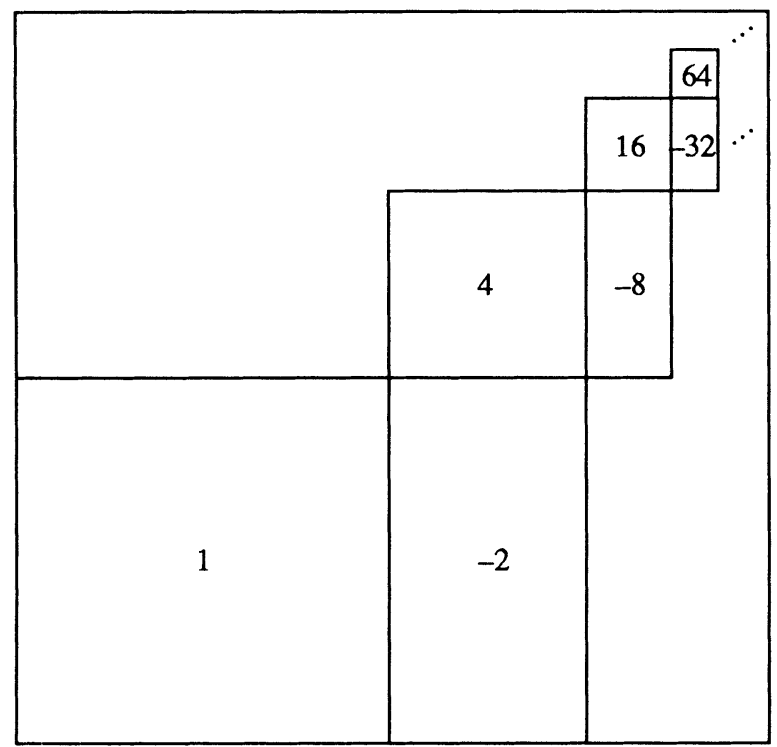

If the function is bounded but the domain is unbounded a similar problem arises: consider

$$
f(x, y)= \begin{cases}1, & \text { if }\lfloor x\rfloor=\lfloor y\rfloor \text { and } x, y \geq 0 \\ -1, & \text { if }\lfloor x\rfloor=\lfloor y+1\rfloor \text { and } x, y \geq 0 \\ 0, & \text { otherwise. }\end{cases}
$$

In order to avoid situations like these we will require that our functions be nonnegative. (Actually, for finite domains all our results will still be valid for functions which are bounded in one direction.)

Since we will want iterated integrals to be equal when they exist we must reckon with another phenomenon, first observed by Sierpinski in 1920: if the Continuum Hypothesis (henceforth referred to as $\mathrm{CH}$ ) holds, then there is a subset of $\mathbf{R}^{2}$ which is countable on every horizontal line and co-countable on every vertical line. (Proof: If $\mathrm{CH}$ holds, there is a well-ordering $\prec$ of $\mathbf{R}$ of order type $\aleph_{1}$; the set of $(x, y)$ with $x \prec y$ in this ordering is countable for fixed $y$ and co-countable for fixed $x$ ). If $f: \mathbf{R}^{2} \rightarrow\{0,1\}$ is the characteristic function of this set, then clearly $\int_{0}^{1} \int_{0}^{1} f d x d y=\int_{0}^{1} 0 d y=0$, while $\int_{0}^{1} \int_{0}^{1} f d y d x=$ $\int_{0}^{1} 1 d x=1$, because countable sets have measure 0 .

Thus, even when we require $f$ nonnegative, iterated integrals can disagree if $\mathrm{CH}$ holds. The full strength of $\mathrm{CH}$ is not needed: if $\kappa$, the cardinality of the smallest nonmeasurable set, equals $c=2^{\aleph_{0}}$, the above construction will still work. By Gödel's result that $\mathrm{CH}$ is consistent with the ZFC system of settheoretical axioms (and we always work within ZFC), there is no hope of proving "strong Fubini theorems" implying that iterated integrals of bounded functions never disagree. Instead we will find conditions, necessarily independent of ZFC, which will imply strong Fubini theorems. 
The simplest statement of a strong Fubini theorem is

$\left(A_{2}\right)$ If $f: \mathbf{R}^{2} \rightarrow \mathbf{R}$ is nonnegative and the iterated integrals $\iint f d x d y$ and $\iint f d y d x$ exist, then these integrals are equal.

Let $\kappa$ be the cardinality of the smallest nonmeasurable set. By the above example, $A_{2}$ implies $\kappa<c$ and so is independent of ZFC. Let $\lambda$ be the cardinality of the smallest union of measure- 0 sets which covers $\mathbf{R}$. Clearly $\aleph_{0}<\kappa, \lambda \leq c=2^{\aleph_{0}}$. One of our results will be as follows:

Theorem. $\kappa<\lambda$ implies $A_{2}$.

In this paper we will find necessary conditions and sufficient conditions for various generalizations of $A_{2}$. These conditions will all follow from the assumption "A real-valued measure on $[0,1]$ exists" and will be consistent with ZFC.

\section{Definitions AND STATEMENT OF RESUlts}

Definition. $\left(*_{n}\right)$ is the following statement:

$\left(*_{n}\right)$ There exist cardinal numbers $\kappa_{1}, \kappa_{2}, \ldots, \kappa_{n}$ such that $\kappa_{1}$ is the cardinality of a nonmeasurable set of real numbers, and for $i=2,3, \ldots, n \kappa_{i}$ is the cardinality of a set of reals which is not the union of $\kappa_{i-1}$ measure- 0 sets.

Note that $\left(*_{2}\right)$ is just the condition $\kappa<\lambda$ above. The $\left(*_{n}\right)$ are all consistent with ZFC; by Theorem 3.18 of Kunen [1984] it is consistent to suppose that $c>\aleph_{\omega}$ and that there is a set $X$ of reals of cardinality $c$ which has countable intersection with every measure- 0 set. In this case, evidently, $\left(*_{n}\right)$ is true for all $n$, taking $\kappa_{i}=\aleph_{i}$ for each $i$, and using subsets of $X$ of cardinality $\kappa_{i}$. Kunen's theorem involves "forcing with $\aleph_{\omega}$ random reals".

Definition. $A_{n}$ is the following statement:

$\left(A_{n}\right)$ If $f: \mathbf{R}^{n} \rightarrow \mathbf{R}$ is nonnegative and all $n$ ! of the iterated integrals

$$
I_{\sigma}=\iint \cdots \int f\left(x_{1}, x_{2}, \ldots, x_{n}\right) d x_{\sigma(1)} d x_{\sigma(2)} \cdots d x_{\sigma(n)}, \quad \sigma \in S_{n},
$$

exist, then those integrals are equal.

$A_{n}$ is a straightforward generalization of $A_{2}$.

Definition. $B_{n}$ is the following statement:

$\left(B_{n}\right)$ If $f: \mathbf{R}^{n} \rightarrow \mathbf{R}$ is nonnegative and any two of the iterated integrals $I_{\sigma}$, $\sigma \in S_{n}$, exist, then those two integrals are equal.

$B_{n}$ is a stronger generalization. Clearly $A_{2}$ and $B_{2}$ say the same thing. Our central theorem is the following:

Theorem 1. $\left(*_{n}\right)$ implies $B_{n}$.

The $\left(*_{n}\right)$ are the "cardinal conditions" in the title of this paper. The $B_{n}$ are the "strong Fubini theorems." We will also prove that $A_{2} \Leftrightarrow A_{n}$ for $n>2$, so the 2-cardinal condition $\left(*_{2}\right)$ implies the $n$-dimensional statement $A_{n}$; the $B_{n}$ seem to be inequivalent and to require $c \geq \aleph_{n}$, but whether the $*_{n}$ are 
necessary as well as sufficient for the $B_{n}$ is open. By Sierpinski's example, the condition $\kappa<c$ is necessary for $B_{2}$, and we will also see that it is necessary that $\alpha<\lambda$, where $\alpha$ is the additivity of Lebesgue measure and $\lambda$ is the "covering number" of Lebesgue measure, as defined earlier.

To state our second main theorem we will need some definitions.

Definition. Let $S$ be an infinite set. A measure on $S$ is a nonnegative realvalued function $\mu$ defined on the subsets of $S$ such that

(i) $\mu(\varnothing)=0$ and $\mu(S)=1$.

(ii) For all $x \in S, \mu(\{x\})=0$.

(iii) If $X_{1}, X_{2}, \ldots$ are pairwise disjoint, then

$$
\mu\left(\bigcup_{n=1}^{\infty} X_{n}\right)=\sum_{n=1}^{\infty} \mu\left(X_{n}\right) .
$$

(Some authors refer to $\mu$ as a "measure with domain $\mathscr{P}(S)$ ".)

Definition. A measure $\mu$ on a set $S$ is atomless if every set $X \subseteq S$ of positive measure can be split into two disjoint sets of positive measure:

(iv) $\mu(X)>0$ implies $X=Y \cup Z$ with $\mu(Y)>0, \mu(Z)>0$, and $Y \cap Z=\varnothing$.

Definition. $\kappa$ is a real-valued measurable cardinal if an atomless measure $\mu$ exists on the cardinal $\kappa$, such that $\mu$ is also $\kappa$-additive: the union of less than $\kappa$ sets of measure 0 is also measure 0 .

Ulam [1930] showed that if an atomless measure exists on any set, then an atomless measure exists on $[0,1]$ and furthermore a real-valued measurable cardinal exists. Although Lebesgue measure satisfies the conditions (i)-(iv) it is not defined on all subsets of $[0,1]$. A real-valued measurable cardinal with an atomless measure exists if and only if Lebesgue measure can be extended to a countably additive measure defined on all subsets of $[0,1]$.

Definition. RVM is the axiom "there exists a measure on $[0,1]$."

This is sometimes stated "the continuum is real-valued measurable", hence the abbreviation RVM; however, the terminology is confusing because all that is implied is that there exists a real-valued measurable cardinal $\leq c$. A measure on $[0,1]$ must be atomless, and conversely, real-valued measurable cardinals with atomless measures must be $\leq c$. Measures with atoms imply the existence of a measurable cardinal; see below.

Real-valued measurable cardinals are weakly inaccessible (an uncountable cardinal is weakly inaccessible iff for all $\lambda<\kappa, \kappa \neq \lambda^{+}$and $\kappa$ is not the union of $\lambda$ cardinals less than $\kappa$ ), and by Gödel's results cannot be shown to exist within ZFC. Hence RVM is a genuinely new axiom.

Solovay [1971] showed that RVM is consistent if and only if the axiom "there exists a measurable cardinal" is consistent; a measurable cardinal $\kappa$ is a cardinal $\kappa$ with a $\kappa$-additive measure taking only the values 0 and 1 , and the existence of a measurable cardinal is a standard "large cardinal axiom". 
Theorem 2. $R V M$ implies $\left(*_{n}\right)$ for all $n$.

It is interesting to contrast Theorem 2, which implies that if Lebesgue measure can be extended to all sets of reals then a strong Fubini theorem holds, with Sierpinski's example which shows that if Lebesgue measure is already defined for all small sets of reals then the strong Fubini theorem fails. In other words, if Lebesgue measure is already defined on too many sets, then it cannot be extended to all sets.

This is not as counterintuitive as it might appear. Vitali's famous construction of a nonmeasurable set used the translation-invariance of Lebesgue measure; any measure on all sets of reals will not be invariant, so having too many sets Lebesgue measurable might well preclude a noninvariant extension.

We will prove several other interesting results along the way; further work and open questions will be discussed in the last section.

\section{Proof of Theorem 1}

We will first prove a useful lemma, originally shown by Freiling [1986] in the 2-dimensional case. Our $n$-dimensional proof is a direct generalization of Freiling's argument, with some inaccuracies corrected.

Lemma. Suppose the iterated integrals

$$
\iint \cdots \int f\left(x_{1}, x_{2}, \ldots, x_{n}\right) d x_{1} d x_{2} \cdots d x_{n}
$$

and

$$
\iint \cdots \int f\left(x_{1}, x_{2}, \ldots, x_{n}\right) d x_{\sigma(1)} d x_{\sigma(2)} \cdots d x_{\sigma(n)}
$$

of a nonnegative function $f$ on $\mathbf{R}^{n}$ exist and are unequal. Then there exists a set $D \subset \mathbf{R}^{n}$ such that

$$
\begin{gathered}
\tilde{\forall} x_{1} \tilde{\forall} x_{2} \ldots \tilde{\forall} x_{n}\left(x_{1}, \ldots, x_{n}\right) \in D, \\
\tilde{\forall} x_{\sigma(1)} \tilde{\forall} x_{\sigma(2)} \ldots \tilde{\forall} x_{\sigma(n)}\left(x_{1}, \ldots, x_{n}\right) \notin D,
\end{gathered}
$$

where the quantifier $\tilde{\forall}$ means "for almost all", that is, except for a set of Lebesgue measure 0.

Remarks. The set $D$ is a generalized "Sierpinski example". The lemma allows us to replace statements about integrals by statements about measure- 0 sets. In this lemma $\mathbf{R}^{n}$ can actually be replaced by any $n$-fold product of separable measure spaces in the hypothesis and any $n$-fold product of separable sufficiently large measure spaces in the conclusion ("sufficiently large" means containing a subset measure-isomorphic to an interval).

Proof. If iterated integrals disagree for a nonnegative function $f: \mathbf{R}^{n} \rightarrow \mathbf{R}$, then they already disagree for the restriction of $f$ to some $n$-cube: apply the monotone convergence theorem $n$ times to show that

$$
\int_{\mathbf{R}} \cdots \int_{\mathbf{R}} f d x_{1} \cdots d x_{n}=\lim _{m \rightarrow \infty} \int_{-m}^{m} \cdots \int_{-m}^{m} f d x_{1} \cdots d x_{n} .
$$


Without loss of generality, therefore, we may assume $f$ is supported on an $n$-cube. We may similarly assume that $f$ is bounded, using the monotone convergence theorem on the sequence of "cut-off" functions $\{\operatorname{Min}(f, 1)$, $\operatorname{Min}(f, 2), \ldots\}$. In fact, we may assume we have a function $f:[0,1]^{n} \rightarrow$ $[0,1]$, such that

$$
\int_{0}^{1} \cdots \int_{0}^{1} f d x_{1} \cdots d x_{n}<\gamma<\int_{0}^{1} \cdots \int_{0}^{1} f d x_{\sigma(1)} \cdots d x_{\sigma(n)},
$$

for some real number $\gamma$. Let us abbreviate $[0,1]$ by $I$.

Now consider the product space $I^{\omega}=\left\{\left\langle x_{1} x_{2} \cdots\right\rangle \mid x_{i} \in I\right\}$, the space of sequences from $I$, with the product topology and the product measure. Define $A \subset\left(I^{\omega}\right)^{n}$ as follows: $\left(\bar{x}_{1}, \bar{x}_{2}, \ldots, \bar{x}_{n}\right) \in A$ iff $\bar{x}_{i}=\left\langle x_{i 1} x_{i 2} \cdots\right\rangle$ and

$$
(\exists M)(\forall k>M) \sum_{j=1}^{k} \frac{1}{k} f\left(x_{1 j}, \ldots, x_{n j}\right) \leq \gamma .
$$

In other words, a point in $\left(I^{\omega}\right)^{n}$ is in $A$ if the seqence of points in $I^{n}$ that it represents has a sequence of $f$-values with upper density at most $\gamma$.

We now give a version of the Law of Large Numbers, adapted from Shiryayev [1984] with the notation "unraveled".

LLN: If the sequence of functions $f_{1}, f_{2}, \ldots:[0,1] \rightarrow \mathbf{R}$ satisfies

(i) $\forall i \int_{0}^{1} f_{i}(t) d t$ exists (expectation exists), and

(ii) $\exists M \forall i \int_{0}^{1}\left(f_{i}(t)-\left(\int_{0}^{1} f_{i}(u) d u\right)\right)^{2} d t<M$ (bounded variances), then for almost all sequences $\bar{t}=\left\langle t_{1} t_{2} \cdots\right\rangle \in I^{\omega}$,

$$
\lim _{n \rightarrow \infty} \frac{1}{n}\left(\sum_{i=1}^{n} f_{i}\left(t_{i}\right)-\sum_{i=1}^{n} \int_{0}^{1} f_{i}(x) d x\right)=0 .
$$

For almost every $t \in[0,1], \int \cdots \int f\left(t, x_{2}, \ldots, x_{n}\right) d x_{2} \cdots d x_{n}$ exists. Therefore, for almost every $\left\langle t_{1} t_{2} \cdots\right\rangle \in I^{\omega}, \int \cdots \int f\left(t_{i}, x_{2}, \ldots, x_{n}\right) d x_{2} \cdots d x_{n}$ exists on each $t_{i}$. For each such $\left\langle t_{1} t_{2} \cdots\right\rangle$, the set

$$
\begin{aligned}
\left\{\bar{u} \in I^{\omega} \mid \lim _{m \rightarrow \infty} \frac{1}{m}(\right. & \sum_{i=1}^{m} \int \cdots \int f\left(t_{i}, u_{i}, x_{3}, \ldots, x_{n}\right) d x_{3} \cdots d x_{n} \\
& \left.\left.-\sum_{i=1}^{m} \int \cdots \int f\left(t_{i}, x_{2}, x_{3}, \ldots, x_{n}\right) d x_{2} d x_{3} \cdots d x_{n}\right)=0\right\}
\end{aligned}
$$

has measure 1, using boundedness of $f$ and applying LLN to the sequence of functions

$$
f_{i}(u)=\int \cdots \int f\left(t_{i}, u, x_{3}, \ldots, x_{n}\right) d x_{3} \cdots d x_{n} .
$$


By LLN again, the set

$$
\begin{aligned}
\left\{\bar{t} \in I^{\omega} \mid \lim _{m \rightarrow \infty} \frac{1}{m}\left(\sum_{i=1}^{m} \int \cdots \int f\left(t_{i}, x_{2}, \ldots, x_{n}\right) d x_{2} \ldots d x_{n}\right)\right. \\
\left.=\int \cdots \int f\left(x_{1}, \ldots, x_{n}\right) d x_{1} \cdots d x_{n}\right\}
\end{aligned}
$$

has measure 1 , using the sequence of functions

$$
f_{i}(t)=\int \cdots \int f\left(t, x_{2}, \ldots, x_{n}\right) d x_{2} \cdots d x_{n}
$$

(that is, the same function for all $i$ ).

Combining these, we get the following:

$$
\begin{array}{r}
\left(\tilde{\forall} \bar{t} \in I^{\omega}\right)\left(\tilde{\forall} \bar{u} \in I^{\omega}\right)\left(\lim _{m \rightarrow \infty} \frac{1}{m}\left(\sum_{i=1}^{m} \int \cdots \int f\left(t_{i}, u_{i}, x_{3}, \ldots, x_{n}\right) d x_{3} \cdots d x_{n}\right)\right. \\
\left.=\int \cdots \int f d x_{1} \cdots d x_{n}\right),
\end{array}
$$

where the quantifier $\tilde{\forall}$ again means "for almost all".

In the general inductive step, we obtain

$$
\begin{array}{r}
\left(\tilde{\forall} \bar{t}_{1} \cdots \tilde{\forall} \bar{t}_{r}\right)\left(\lim _{m \rightarrow \infty} \frac{1}{m}\left(\sum_{i=1}^{m} \int \cdots \int f\left(t_{1 i}, \ldots, t_{r i}, x_{r+1}, \ldots, x_{n}\right) d x_{r+1} \cdots d x_{n}\right)\right. \\
\left.=\int \cdots \int f d x_{1} \cdots d x_{n}\right),
\end{array}
$$

ultimately arriving at

$$
\begin{aligned}
& \left(\tilde{\forall} \bar{t}_{1} \in I^{\omega}\right)\left(\tilde{\forall} \bar{t}_{2} \in I^{\omega}\right) \cdots\left(\tilde{\forall} \bar{t}_{n} \in I^{\omega}\right) \\
& \quad\left(\lim _{m \rightarrow \infty} \frac{1}{m}\left(\sum_{i=1}^{m} f\left(t_{1 i}, t_{2 i}, \ldots, t_{n i}\right)\right)=\int \cdots \int f d x_{1} d x_{2} \ldots d x_{n}\right) .
\end{aligned}
$$

But since the value of the iterated integral at the right is a real number less than $\gamma$, all the points that satisfy the condition are in $A \subset\left(I^{\omega}\right)^{n}$. Therefore, we have the familiar-looking fact

$$
\tilde{\forall} \bar{t}_{1} \tilde{\forall} \bar{t}_{2} \ldots \tilde{\forall}_{n}\left(\bar{t}_{1}, \bar{t}_{2}, \ldots, \bar{t}_{n}\right) \in A \text {. }
$$

Let $\Phi: I^{\omega} \rightarrow \mathbf{R}$ be a bijection which preserves measure-0 sets (these are easy to construct between any two separable sufficiently large measure spaces-e.g. use $2^{\omega} \cong I$ and $2^{\omega} \cong\left(2^{\omega}\right)^{\omega}$ to get $I \cong I^{\omega}$, etc. $)$. Define $\left(x_{1}, \ldots, x_{n}\right) \in \mathbf{R}^{n}$ to be in $D$ if $x_{i}=\Phi\left(\bar{t}_{i}\right)$ and $\left(\bar{t}_{1}, \ldots, \bar{t}_{n}\right) \in A$. Then clearly

$$
\tilde{\forall} x_{1} \ldots \tilde{\forall} x_{n}\left(x_{1}, \ldots, x_{n}\right) \in D \text {. }
$$

This is just condition (1) of the conclusion of the lemma. By doing the whole argument over, we obtain $\tilde{\forall} x_{\sigma(1)} \ldots \tilde{\forall} x_{\sigma(n)}\left(x_{1}, \ldots, x_{n}\right) \notin D$, which is condition (2) of the conclusion. 
Theorem 1. $\left(*_{n}\right)$ implies $B_{n}$.

Suppose $\kappa_{1}, \ldots, \kappa_{n}$ are cardinals with $\kappa_{1}$ the cardinality of a nonmeasurable set of reals and $\kappa_{i}, i=2,3, \ldots, n$, the cardinality of a set of reals which is not the union of $\kappa_{i-1}$ measure-0 sets. Then whenever $f: \mathbf{R}^{n} \rightarrow \mathbf{R}$ is nonnegative and the iterated integrals $\int \cdots \int f d x_{1} \cdots d x_{n}$ and $\int \cdots \int f d x_{\sigma(1)} \cdots d x_{\sigma(n)}$ exist, those integrals are equal.

Proof. Suppose the contrary, that the hypothesis is true and the conclusion false. Since the hypothesis is true, we have sets $A_{1}, A_{2}, \ldots, A_{n} \subset \mathbf{R}$ with $\left|A_{i}\right|=\kappa_{i}, A_{1}$ nonmeasurable, and $A_{i}$ not the union of $\kappa_{i-1}$ measure-0 sets for $i=2,3, \ldots, n$. Since the conclusion is false, applying the Lemma we obtain a set $D \subset \mathbf{R}^{n}$ such that

(1) $\tilde{\forall} x_{1} \cdots \tilde{\forall} x_{n}\left(x_{1}, \ldots, x_{n}\right) \in D$;

(2) $\tilde{\forall} x_{\sigma(1)}, \ldots \tilde{\forall} x_{\sigma(n)}\left(x_{1}, \ldots, x_{n}\right) \notin D$.

Given $n-1$ real numbers $t_{1}, \ldots, t_{n-1}$ we will construct a set

$$
B\left(t_{1}, \ldots, t_{n-1}\right) \subset \mathbf{R}
$$

consisting of all the "bad points" associated with $t_{1}, \ldots, t_{n-1}$.

Construction. Step 1. Let

$$
B_{1}\left(t_{1}, \ldots, t_{n-1}\right)=\left\{x \mid \tilde{\forall} x_{2} \tilde{\forall} x_{3} \ldots \tilde{\forall} x_{n}\left(x, x_{2}, x_{3}, \ldots, x_{n}\right) \notin D\right\} .
$$

$B_{1}$ is the same for all $\left(t_{1}, \ldots, t_{n-1}\right)$ and has measure 0 because it consists of the "bad points" for the first quantifier in condition (1).

Step 2. Let $B_{2}\left(t_{1}, \ldots, t_{n-1}\right)=\left\{x \mid \tilde{\forall} x_{3} \ldots \tilde{\forall} x_{n}\left(t_{1}, x, x_{3}, \ldots, x_{n}\right) \notin D\right\}$ unless $t_{1} \in B_{1}$, in which case $B_{2}\left(t_{1}, \ldots, t_{n-1}\right)=\varnothing . B_{2}$ depends only on $t_{1}$ and has measure 0 because it consists of the bad points for the second quantifier in condition (1), given $x_{1}=t_{1}$ and $t_{1}$ is not already bad (if $t_{1}$ is already bad the second "almost all" quantifier is irrelevant and $B_{2}$ has measure 0 because it is empty).

Step $k$. Let

$B_{k}\left(t_{1}, \ldots, t_{n-1}\right)=\left\{x \mid \tilde{\forall} x_{k+1} \ldots \tilde{\forall} x_{n}\left(t_{1}, t_{2}, \ldots, t_{k-1}, x, x_{k+1}, \ldots, x_{n}\right) \notin D\right\}$

unless $t_{i} \in B_{i}$ for some $i<k$, in which case $B_{k}\left(t_{1}, \ldots, t_{k-1}\right)=\varnothing$. $B_{k}$ depends on $t_{1}, \ldots, t_{k-1}$ and has measure 0 because when it is nonempty it consists of the bad points for the $k$ th quantifier in condition (1), given $x_{1}=$ $t_{1}, \ldots, x_{k-1}=t_{k-1}$.

Step $n$. Finally, let $B_{n}\left(t_{1}, \ldots, t_{n-1}\right)=\left\{x \mid\left(t_{1}, \ldots, t_{n-1}, x\right) \notin D\right\}$ unless $t_{i} \in B_{i}$ for some $i<n$, in which case $B_{n}=\varnothing$. As above, $B_{n}$ has measure 0 .

We now repeat the construction for condition (2). 
Step $1^{\prime}$. Let

$$
\begin{aligned}
B_{1}^{\prime}\left(t_{1}, \ldots, t_{n-1}\right) & \\
& =\left\{x \mid \tilde{\forall} x_{\sigma(2)} \ldots \tilde{\forall} x_{\sigma(n)}\left(x_{1}, \ldots, x_{\sigma(1)-1}, x, x_{\sigma(1)+1}, \ldots, x_{n}\right) \in D .\right.
\end{aligned}
$$

$B_{1}^{\prime}$ is the same for all $\left(t_{1}, \ldots, t_{n-1}\right)$ and has measure 0 because it consists of the bad points for the first quantifier in condition (2).

Step $k^{\prime}$. In general, $B_{k}^{\prime}$ consists of

$$
\left\{x \mid \tilde{\forall} x_{\sigma(k+1)} \cdots \tilde{\forall} x_{\sigma(n)} \vec{p} \in D\right\},
$$

where $\vec{p}$ is the $n$-tuple with $t_{i}$ in the $i$ th place for $i<k, x$ in the $\sigma(k)$ th place, and $x_{\sigma(i)}$ in the $\sigma(i)$ th place for $i>k$, unless $t_{j} \in B_{j}^{\prime}$ for some $j<k$, in which case $B_{k}^{\prime}$ is empty. $B_{k}^{\prime}$ has measure 0 as above.

Define $B\left(t_{1}, \ldots, t_{n-1}\right)=\bigcup_{i=1}^{n}\left(B_{i}\left(t_{1}, \ldots, t_{n-1}\right) \cup B_{i}^{\prime}\left(t_{1}, \ldots, t_{n-1}\right)\right)$.

The key facts about $B$ are these:

(i) $B\left(t_{1}, \ldots, t_{n-1}\right)$ has measure 0 .

(ii) For all $\left(z_{1}, \ldots, z_{n}\right) \in \mathbf{R}^{n}$, there is an $i$ such that

$$
z_{i} \in B\left(z_{1}, z_{2}, \ldots, z_{i-1}, z_{i+1}, \ldots, z_{n}\right) .
$$

The first fact is obvious, because $B$ is the union of $2 n$ measure- 0 sets. To see the second fact, note that either $\left(z_{1}, z_{2}, \ldots, z_{n}\right) \notin D$ or $\left(z_{1}, z_{2}, \ldots, z_{n}\right) \in$ $D$. In the first case, one of the quantifiers in condition (1) must not apply, so either $z_{1} \in B_{1}, z_{2} \in B_{2}, \ldots$, or $z_{n} \in B_{n}$. In the second case, one of the quantifiers in condition (2) must not apply, so either $z_{\sigma(1)} \in B_{1}^{\prime}, z_{\sigma(2)} \in$ $B_{2}^{\prime}, \ldots$, or $z_{\sigma(n)} \in B_{n}^{\prime}$.

Now recall the sets $A_{1}, A_{2}, \ldots, A_{n}$ with $\left|A_{i}\right|=\kappa_{i}, A_{1}$ nonmeasurable, and $A_{i}$ not the union of $\kappa_{i-1}$ measure-0 sets for $i=2,3, \ldots, n$. We may suppose $A_{1} \subset A_{2} \subset \cdots \subset A_{n}$ (for example, by redefining $A_{2}^{\prime}=A_{1} \cup A_{2}$, $A_{3}^{\prime}=A_{1} \cup A_{2} \cup A_{3}$, etc.). We will construct a point $\left(z_{1}, \ldots, z_{n}\right) \in \mathbf{R}^{n}$ with $z_{i} \in A_{i}$. In what follows, $\vec{t}$ will denote an $(n-1)$-tuple $\left(t_{1}, t_{2}, \ldots, t_{n-1}\right)$ and $B(\vec{t})$ will be as defined above.

Construction. Pick

$$
z_{n} \in A_{n} \backslash \bigcup_{\vec{t} \in\left(A_{n-1}\right)^{n-1}} B(\vec{t}) .
$$

This is possible because $\left|\left(A_{n-1}\right)^{n-1}\right|=\kappa_{n-1}$ and $A_{n}$ is not the union of $\kappa_{n-1}$ measure- 0 sets.

Pick

$$
z_{n-1} \in A_{n-1} \backslash \bigcup_{\vec{t} \in\left(A_{n-2}\right)^{n-2} \times\left\{z_{n}\right\}} B(\vec{t}) .
$$

This is possible because $\left|\left(A_{n-2}\right)^{n-2}\right|=\kappa_{n-2}$ and $A_{n-1}$ is not the union of $\kappa_{n-2}$ measure- 0 sets. 
Pick

$$
z_{n-2} \in A_{n-2} \backslash \bigcup_{\vec{t} \in\left(A_{n-3}\right)^{n-3} \times\left\{z_{n-1}\right\} \times\left\{z_{n}\right\}} B(\vec{t}) .
$$

Pick

$$
z_{2} \in A_{2} \backslash \bigcup_{\vec{t} \in A_{1} \times\left\{z_{3}\right\} \times\left\{z_{4}\right\} \times \cdots \times\left\{z_{n}\right\}} B(\vec{t}) .
$$

This is possible since $\left|A_{1}\right|=\kappa_{1}$ and $A_{2}$ is not a union of $\kappa_{1}$ measure- 0 sets. Finally, pick $z_{1} \in A_{1} \backslash B\left(z_{2}, z_{3}, \ldots, z_{n}\right)$.

This is possible because $A_{1}$ is nonmeasurable and so is not contained in the measure-0 set $B\left(z_{2}, \ldots, z_{n}\right)$.

By construction, $z_{i} \in A_{i}$ and therefore

$$
z_{i} \notin B\left(z_{1}, z_{2}, \ldots, z_{i-1}, z_{i+1}, \ldots, z_{n}\right)
$$

for all $i$. But this contradicts the second fact about the function $B$.

We have just shown that the cardinal condition $\left(*_{n}\right)$ is sufficient for the strong Fubini theorem $B_{n}$. Whether it is necessary seems a more difficult question. It is reasonable to conjecture that $B_{n+1}$ is strictly stronger than $B_{n}$ for all $n$. Surprisingly, this is not the case for the weaker statements $A_{n}$.

Theorem. For all $n, A_{2} \Leftrightarrow A_{n}$.

Proof. $A_{n}$ implies $A_{2}$ is trivial. Assume $A_{2}$, and by induction assume $A_{n-1}$ is true. Consider now the $n$ ! integrals

$$
I_{\sigma}=\iint \cdots \int f\left(x_{1}, x_{2}, \ldots, x_{n}\right) d x_{\sigma(1)} d x_{\sigma(2)} \cdots d x_{\sigma(n)},
$$

for $\sigma \in S_{n}$. Suppose $\sigma_{1}$ and $\sigma_{2}$ are two permutations which satisfy $\sigma_{1}(n)=$ $\sigma_{2}(n)$. Then we can apply $A_{n-1}$ and conclude that the inner $(n-1)$-fold integrals are equal, so $I_{\sigma_{1}}=I_{\sigma_{2}}$.

Now suppose $\sigma_{1}$ and $\sigma_{2}$ are two permutations which satisfy $\sigma_{1}(1)=\sigma_{2}(1)$. Then, replacing $f\left(x_{1}, \ldots, x_{n}\right)$ by

$$
\left[\int f\left(x_{1}, \ldots, x_{n}\right) d x_{\sigma_{1}(1)}\right]=\left[\int f\left(x_{1}, \ldots, x_{n}\right) d x_{\sigma_{2}(1)}\right]
$$

we can apply $A_{n-1}$ again and conclude that the outer $(n-1)$-fold integrals are equal, so that $I_{\sigma_{1}}=I_{\sigma_{2}}$.

Since any permutation in $S_{n}, n \geq 3$, can be expressed as a product of permutations which fix 1 and permutations which fix $n$, we must have $I_{\sigma_{1}}=I_{\sigma_{2}}$ for all $\sigma_{1}, \sigma_{2} \in S_{n}$.

The reason the proof does not extend to show $B_{2}$ implies $B_{n}$ is that the "connecting" permutations may not have their integrals exist.

We have seen that " $\kappa<\lambda$ " (which is our condition $\left(*_{2}\right)$ ) is a sufficient condition for $B_{2}$. We have also seen that " $\kappa<c$ " is a necessary condition. Another necessary condition is given by the next result. 
Theorem. Let $\alpha$ be the additivity of Lebesgue measure: any union of $<\alpha$ sets of measure 0 has measure 0 . Let $\lambda$ be the covering number of Lebesgue measure as defined in the introduction. (Clearly $\alpha \leq \lambda$.) If $\alpha=\lambda$, then $B_{2}$ is false (so $\alpha<\lambda$ is a necessary condition for $B_{2}$ ).

Proof. Suppose $\alpha=\lambda$. Let $A_{\gamma}, \gamma<\lambda$, be a collection of measure-0 sets which covers $\mathbf{R}$; we may assume they are disjoint (e.g., by redefining $A_{\gamma}^{\prime}=$ $\left.A_{\gamma} \backslash \bigcup_{\delta<\gamma} A_{\delta}\right)$. Define $D \subset \mathbf{R}^{2}$ as follows: $(x, y) \in D$ iff $x \in A_{\delta}, y \in A_{\gamma}$, and $\delta<\gamma$. For fixed $y$, the set $\{x \mid(x, y) \in D\}$ is the union of less than $\lambda$ measure0 sets, and so (since $\alpha=\lambda$ ) is measure 0 . For fixed $\mathrm{x}$, the set $\{y \mid(x, y) \in D\}$ is co-measure 0 for the same reason. Thus $D$ is a Sierpinski example and $B_{2}$ is false.

Both of the necessary conditions $\kappa<c$ and $\alpha<\lambda$ follow from the following stronger condition, which is also necessary for $B_{2}$ :

$(\star)$ If $\mathbf{R}$ is a union of $\gamma$ measure- 0 sets, then some union of less than $\gamma$ of them is not measure 0 . That is, if $\mathbf{R}=\bigcup_{\delta<\gamma} X_{\delta}$ with the $X_{\delta}$ measure 0 , then $\bigcup_{\delta<\xi} X_{\delta}$ is not measure 0 for some $\xi<\gamma$.

It is clear that $(\star)$ is indeed necessary for $B_{2}$-the proof above works. Thus we have $\left(*_{2}\right) \rightarrow B_{2} \rightarrow(\star) . \quad(\star)$ is equivalent to Fremlin's condition $A F_{\infty}$ ("if every subcollection of a collection of measure- 0 sets is measurable, then the union of the whole collection has measure 0 "), which he deduced from $\left(*_{2}\right)$ in Fremlin [1987]. In the case $\alpha=\aleph_{1}, \kappa=\lambda=\aleph_{2}, c=\aleph_{3}$, the gap between the necessary and sufficient conditions is clear: $B_{2}$ may be provably true, provably false, or neither (but not both, as these conditions are consistent with ZFC).

Open Question. Is $B_{2}$ true, false, or independent in this case?

Conjecture. $\quad c \geq \aleph_{n}$ is a necessary condition for $B_{n}$ (true if $n=2$ ).

The condition $(\star)$ can be stated more simply " $\mathbf{R}$ is not a chain of measure- 0 sets". If $\mathbf{R}$ is such a chain, we have seen that we can construct a Sierpinski example, but in fact more is true: iterated integrals of bounded functions can be anything at all.

Theorem. If $(\star)$ is false, then for any assignment of values in $[0, \infty]$ to the $n !$ integrals $\int \cdots \int f\left(x_{1}, \ldots, x_{n}\right) d x_{\sigma(1)} \cdots d x_{\sigma(n)}$, there exists a bounded, nonnegative function $g: \mathbf{R}^{n} \rightarrow \mathbf{R}$ with the prescribed values for its integrals.

This is the ultimate generalization of Sierpinski's example. The $\left(*_{n}\right)$ are successively stronger versions of $(\star)$, which rule out these examples in higher and higher dimensions. Actually, negative values for the integrals can also be handled, by splitting the $n$ ! values into "positive and negative parts", in which case $g$ will only be bounded.

Proof. Our function $g$ will actually be the characteristic function of a set, which will be bounded if none of the integrals are $\infty$. We will need a lemma. 
Lemma. Let $v:\{1,2, \ldots, n\} \times \mathbf{R}^{n-1} \rightarrow[0, \infty]$ be defined on the 1-dimensional cross-sections of $\mathbf{R}^{n}$. If $(\star)$ is false, there exists $D \subset \mathbf{R}^{n}$ such that

$$
\left\{x \in \mathbf{R} \mid\left(t_{1}, t_{2}, \ldots, t_{i-1}, x, t_{i+1}, \ldots, t_{n}\right) \in D\right\}
$$

has measure

$$
v\left(i ; t_{1}, t_{2}, \ldots, t_{i-1}, t_{i+1}, \ldots, t_{n}\right) .
$$

That is, we can find a set whose measure on 1-dimensional cross-sections has arbitrarily prescribed values.

Proof of Lemma. ( $(\star)$ is false, so we have a collection of $\gamma$ sets $X_{\xi}, \xi<\gamma$, such that $\bigcup X_{\xi}=\mathbf{R}$ but any union of less than $\gamma$ of them has measure 0 , for some cardinal $\gamma$.

Let $w:\{1,2, \ldots, n\} \times \gamma^{n-1} \rightarrow \gamma$ be a bijection (this exists since $\gamma$ is infinite). The domain of $w$ is the "1-dimensional cross-sections" of the space $\gamma^{n}$. We will define $D$ by transfinite induction in $\gamma$ stages.

At each stage we have an element of dom $w$, which is an $n$-tuple $\left\{i ; \xi_{1}, \xi_{2}\right.$, $\left.\ldots, \xi_{n-1}\right\}$ with $i \in\{1,2, \ldots, n\}$ and the $\xi_{j}$ 's ordinals $<\gamma$. Consider

$$
X_{\xi_{1}} \times X_{\xi_{2}} \times \cdots \times X_{\xi_{i-1}} \times \mathbf{R} \times X_{\xi_{i}} \times \cdots \times X_{\xi_{n-1}} \subset \mathbf{R}^{n}
$$

This is a collection of one-dimensional cross-sections

$$
\left\{t_{1}\right\} \times \cdots \times\left\{t_{i-1}\right\} \times \mathbf{R} \times\left\{t_{i+1}\right\} \times \cdots \times\left\{t_{n}\right\},
$$

with

$$
t_{j} \in \begin{cases}X_{\xi_{j}}, & \text { if } j<i, \\ X_{\xi_{j-1}}, & \text { if } i<j .\end{cases}
$$

On each one of these cross-sections, define

$\left(t_{1}, \ldots, t_{i-1}, x, t_{i+1}, \ldots, t_{n}\right) \in D \Leftrightarrow x \in\left[0, v\left(i ; t_{1}, \ldots, t_{i-1}, t_{i+1}, \ldots, t_{n}\right)\right)$,

unless the point $\left(t_{1}, \ldots, t_{i-1}, x, t_{i+1}, \ldots, t_{n}\right)$ has already been defined as not being in $D$ at an earlier stage. Since there have been fewer than $\gamma$ earlier stages, and each stage only affected, in each one of these cross-sections, a set of points equal to a subset of an $X_{\xi}$, only a measure-0 set of points has been affected, so the measure of $D$ on the cross-sections is as desired. Because of the "unless", these cross-sections will not be affected by later stages; the reason each earlier stage affected at most an $X_{\xi}$ in each cross-section is that the intersection with the cross-sections of that stage corresponds to at most a single point in the quotient space $\gamma^{n}$.

Now that we can define the measure of $D$ arbitrarily on 1-dimensional crosssections, we can prove the theorem. For each $\sigma \in S_{n}$, let $f(\sigma)$ be the desired value of the iterated integral

$$
\int \cdots \int g\left(x_{1}, \ldots, x_{n}\right) d x_{\sigma(1)} \cdots d x_{\sigma(n)},
$$

with $f(\sigma) \in[0, \infty]$. 
By induction, we may assume that for the subset of permutations with $\sigma(1)=$ $i$, we have a bounded function $G_{i}: \mathbf{R}^{n-1} \rightarrow \mathbf{R}$ with

$$
\int \cdots \int g_{i}\left(x_{1}, x_{2}, \ldots, x_{i-1}, x_{i+1}, \ldots, x_{n}\right) d x_{\sigma(2)} \cdots d x_{\sigma(n)}=f(\sigma),
$$

for each $i$.

Let $D \subset \mathbf{R}^{n}$ be such that the measure of $D$ on the 1-dimensional crosssection

$$
\left\{t_{1}\right\} \times \cdots \times\left\{t_{i-1}\right\} \times \mathbf{R} \times\left\{t_{i+1}\right\} \times \cdots \times\left\{t_{n}\right\}
$$

is $g_{i}\left(t_{1}, t_{2}, \ldots, t_{i-1}, t_{i+1}, \ldots, t_{n}\right)$ for all $\left(i ; t_{1}, \ldots, t_{i-1}, t_{i+1}, \ldots, t_{n}\right)$. Let $g$ be the characteristic function of $D$. Then

$$
\begin{aligned}
\int \cdots & \int g\left(x_{1}, \ldots, x_{n}\right) d x_{\sigma(1)} \ldots d x_{\sigma(n)} \\
& =\int \cdots \int\left[\int g\left(x_{1}, \ldots, x_{n}\right) d x_{\sigma(1)}\right] d x_{\sigma(2)} \ldots d x_{\sigma(n)} \\
& =\int \cdots \int g_{i}\left(x_{1}, \ldots, x_{i-1}, x_{i+1}, \ldots, x_{n}\right) d x_{\sigma(2)} \ldots d x_{\sigma(n)} \\
& =f(\sigma)
\end{aligned}
$$

as desired.

To sum up: in this section we have found sufficient conditions $\left(*_{n}\right)$ for the strong Fubini theorems $B_{n}$, shown that the somewhat less strong Fubini theorems $A_{n}$ are all equivalent to $B_{2}$, and seen that the condition $(\star)$ is a necessary condition for $B_{2}$, because if $(\star)$ is false iterated integrals can be anything at all. ( $\star$ ) implies the weaker necessary conditions $\kappa<c$ and $\alpha<\lambda$. It is desirable to narrow the gap between the necessary and sufficient conditions, and to find necessary conditions for $B_{n}$ with $n>2$ (e.g. $c \geq \aleph_{n}$ ).

\section{Proof of Theorem 2}

The existence of a real-valued measurable cardinal (henceforth abbreviated RVM) is equivalent to the existence of a countably additive measure defined on all subsets of $[0,1]$ (see Jech [1978] for details), which can be taken to be an extension of Lebesgue measure.

If there were no nonmeasurable sets, integrals would always exist, so postulating an extension of Lebesgue measure to all sets would seem to be a kind of regularity assumption, making it harder to construct Sierpinski examples and other pathology. It turns out to be the case that Lebesgue measure is much better behaved if RVM is true, even though the extension to all sets given by RVM behaves pathologically (e.g. it is not translation-invariant and admits a Sierpinski example).

We quote a standard result which can be found in Jech [1978]: RVM implies that there exists a measure $\bar{\mu}$ defined on all subsets of $[0,1]$, such that

(i) If $X \subset[0,1]$ is Lebesgue measurable, with measure $\mu(X)$, then $\bar{\mu}(X)=$ $\mu(X)$ (that is, $\bar{\mu}$ is an extension of the Lebesgue measure $\mu$ ). 
(ii) There exists a set $A$, with $\bar{\mu}(A)=1,|A|=\kappa$, and any union of $<\kappa$ sets with $\bar{\mu}$-measure 0 has $\bar{\mu}$-measure 0 .

We can now prove the following:

Theorem 2. Suppose RVM is true. Then for all $n$, there exist cardinals $\kappa_{1}, \ldots$, $\kappa_{n}$ with $\kappa_{1}$ the cardinality of a nonmeasurable set and $\kappa_{i}, i>1$, the cardinality of a set not the union of $\kappa_{i-1}$ measure-0 sets (referring to Lebesgue measure, of course ).

In short, Theorem 2 says "RVM implies $\forall n\left(*_{n}\right)$ ". This theorem is a corollary of the next one:

Theorem. Let $\mu, \bar{\mu}, A$, and $\kappa$ be as above. If $\lambda$ is any cardinal $<\kappa$, then there is a set $X \subset \mathbf{R}$, of cardinality less than $\kappa$, which is not the union of $\lambda$ measure-0 sets (in the sense of Lebesgue measure $\mu$ ). (Remark: This is wellknown in the case $\lambda=1$.)

(To get Theorem 2 from this, let $\lambda_{0}=\aleph_{0}$. Then the theorem gives us a set $X_{1}$ with $\left|X_{1}\right|=\kappa_{1}<\kappa$, which is nonmeasurable since it is not measure 0 and has cardinality less than $c$. Let $\lambda_{1}=\kappa_{1}$ (necessarily larger than $\lambda_{0}$ ), and the theorem gives a set $X_{2}$ with $\left|X_{2}\right|=\kappa_{2}<\kappa$ and $X_{2}$ not the union of $\kappa_{1}$ measure- 0 sets. Repeat until you have enough cardinals. This provides another proof that RVM $\rightarrow c>\aleph_{\omega}$; indeed, it shows that $\kappa$ must be weakly inaccessible, and hence that RVM is not only independent of ZFC, but incapable of being proved consistent.)

Proof. Suppose the theorem is false. Then we have, for every set $X$ with $|X|<$ $\kappa$, an expression of $X$ as a union of $\lambda$ Lebesgue-measure-0 sets: $X=\bigcup_{\xi<\lambda} X_{\xi}$.

Consider a well-ordering $\prec$ of $A$, of type $\kappa$. For each $y \in A$, let $A_{y}$ be the set $\{x \in A \mid x \prec y\}$. The $A_{y}$ 's all have cardinality $<\kappa$, so can be expressed as unions of $\lambda$ Lebesgue-measure- 0 sets:

$$
A_{y}=\bigcup_{\xi<\lambda} A_{y \xi}, \quad \mu\left(A_{y \xi}\right)=0 \text { for all } y, \xi .
$$

Each $A_{y \xi}$ is contained in a Borel set $B_{y \xi}$, with $B_{y \xi}$ being a countable intersection of countable unions of rational intervals:

$$
A_{y \xi} \subset B_{y \xi}=\bigcap_{m=1}^{\infty} \bigcup_{n=1}^{\infty} R_{m n y \xi},
$$

where $R_{m n y \xi}$ is an interval with rational endpoints and $\mu\left(B_{y \xi}\right)=0$.

For a rational interval $J$, define

$$
C_{m n \xi J}=\left\{y \mid R_{m n y \xi}=J\right\} .
$$

That is, $C_{m n \xi J}$ consists of the $y \in A$ such that in the $\xi$ th Borel set containing the $x$ less than $y, J$ is the interval in the $(m, n)$ spot. (Remark: "less than" here always refers to the well-ordering $\prec$, not the standard ordering on $[0,1]$.) 
Now define

$$
E_{\xi}=\bigcap_{m} \bigcup_{n} \bigcup_{J}\left(J \times C_{m n \xi J}\right) .
$$

$E_{\xi}$ is a subset of the unit square $[0,1] \times[0,1]$. For fixed $y, E_{\xi}$ is just the $\xi$ th Borel set covering $A_{y}$, and has measure 0 (both $\mu$ and $\bar{\mu}$ ).

Consider the product measure $\mu \times \bar{\mu}$ on $[0,1] \times[0,1]$. The set $E_{\xi}$ is measurable in the product measure, because it is a countable intersection of countable unions of "rectangles" ( $J$, being an interval, is $\mu$-measurable, and $C_{m n \xi J}$, being a set of reals, is $\bar{\mu}$-measurable).

Since horizontal sections of $E_{\xi}$ have $\mu$-measure $0, \mu$-almost all vertical sections of $E_{\xi}$ have $\bar{\mu}$-measure 0 , by the (real, original, classical) Fubini theorem. Each $E_{\xi}$ has a Lebesgue-measure-0 set of bad points, and since there are only $\lambda$ of these sets of bad points they do not cover $A$ (which we recall cannot be covered by $<\kappa$ sets of $\bar{\mu}$-measure 0 , and so cannot be covered by $<\kappa$ sets of $\mu$-measure 0 because $\bar{\mu}$ is 0 on them too).

Thus, there is a point $x \in A$ which is not a bad point for any $\xi$, which means that for all $\xi,\left\{y \mid(x, y) \in E_{\xi}\right\}$ has $\bar{\mu}$-measure 0 . But $\bar{\mu}$ is $\kappa$-additive, so $\left\{y \mid \exists \xi(x, y) \in E_{\xi}\right\}$ has $\bar{\mu}$-measure 0 .

On the other hand, for any y with $x \prec y, x$ is in $A_{y}$ and so is in one of the Borel sets $B_{y \xi}$ covering $A_{y}$, and so is in $E_{\xi}$ for some $\xi$. This contradicts the previous sentence, because $\{y \mid x \prec y\}$ cannot be contained in a $\bar{\mu}$-measure- 0 set since it has $\bar{\mu}$-measure $\bar{\mu}(A)-\bar{\mu}\left(A_{y}\right)=1-0=1$.

We have seen that RVM $\rightarrow\left(*_{n}\right)$. This theorem is an expression of the general principle that RVM implies most of the properties of random real models; see Gitik and Shelah [1988].

The proof of Theorem 2 incorporates some simplifications due to Fremlin (private communication). Kanamori (private communication) points out that Theorem 2 can also be obtained as a consequence of the $\pi_{1}^{2}$-indescribability of RVM's (see Solovay [1971]); the proof given is direct and does not depend on this technical result. Theorem 1, in the special case $n=2$ only, was proved independently by Laczkovich in 1985 (J. Pawlikowski, private communication). The consistency of the 2-dimensional strong Fubini theorem $B_{2}$ was demonstrated by H. Friedman [1980], before it was shown that $\left(*_{2}\right)$ implies $B_{2}$.

\section{SUMMARY AND OPEN QUESTIONS}

In addition to our two main theorems $\left(\left(*_{n}\right) \rightarrow B_{n}\right.$ and RVM $\left.\rightarrow\left(*_{n}\right)\right)$ we have found various necessary and sufficient conditions for "strong Fubini theorems" and their counterparts, "generalized Sierpinski examples". Of particular interest was the condition

$(\star): \mathbf{R}$ is not a chain of measure- 0 sets,

in the absence of which iterated integrals can be absolutely arbitrary.

The important test case for the gap between necessary and sufficient conditions is the following: 
Open Question 1. Suppose $\alpha=\aleph_{1}, \kappa=\lambda=\aleph_{2}$, and $c=\aleph_{3}$. What can be said about the strong Fubini theorem $B_{2}$ ?

Another question from $\S 3$ was stated as a conjecture:

Conjecture. $c \geq \aleph_{n}$ is a necessary condition for $B_{n}$ (true if $n=2$ ).

One way to generalize the $B_{n}$ is to no longer require that iterated integrals exist, but merely that cross-sections exist. This is clearest in the case $n=2$, when we have the "super-strong Fubini theorem":

$D_{2}$ : If $\forall t \int f(t, y) d y$ and $\int f(x, t) d x$ exist, then the iterated integrals $\iint f d x d y$ and $\iint f d y d x$ exist and are equal.

In another paper I will show $\left(*_{2}\right) \rightarrow D_{2}$; higher-dimensional generalizations are unclear.

Open Question 2. To what extent can the assumptions of iterated integral existence be weakened to the existence of cross-sections in the presence of the axioms $\left(*_{n}\right)$ ? Which sets of cross-sections must exist for two particular iterated integrals to be equal? (If all 1-dimensional cross-sections exist, that is, are Lebesgue measurable, then everything reduces to the 2-dimensional case.)

The existence of a real-valued measurable cardinal is known to have many consequences in analysis. The strong Fubini theorems $B_{n}$ are interesting examples which have an intuitive appeal, and may be more acceptable than the axioms RVM or $\left(*_{n}\right)$. What further consequences do the $B_{n}$ have, in addition to the ones we have already seen such as the existence of small nonmeasurable sets?

One example may be seen from the unlikely source of quantum mechanics. Pitowsky [1983] and Gudder [1984] have developed nonstandard models of spin and phase space for elementary particles, in an attempt to resolve the philosophical and methodological problems of orthodox quantum mechanics. A key feature of these models is that measurement of noncommuting observables corresponds to integrating integrals in different orders, so any requirement that iterated integrals always match is inconsistent with these models. In particular, our statement $B_{3}$ implies that no "spin- $\frac{1}{2}$ functions" of the type envisioned by Pitowsky can exist.

Pitowsky and Gudder found it necessary to assume the Continuum Hypothesis to build their models-the results on the consistency of strong Fubini theorems show that some such ZFC-independent assumption was necessary. Even more interestingly, any physical evidence for their theories (or philosophical evidence, since their motivation was to avoid the philosophical problems of orthodox quantum mechanics) is also evidence against mathematical statements like $\left(*_{n}\right)$; since these statements are independent of ZFC we have a potential new source of mathematical knowledge, as envisioned by Gödel [1964].

I would like to thank Robert Solovay, Aki Kanamori, David Fremlin, Sy Friedman, and Harvey Friedman for their advice and encouragement, and Professor Richard Palais for his patience. 


\section{REFERENCES}

C. Freiling, [1986] Axioms of symmetry: throwing darts at the real number line, J. Symbolic Logic 51 (1986), 190-200.

D. Fremlin, [1983] Cichón's diagram, Séminaire Initiation à l'Analyse 23 (1983/84), no. 5. [1987] Measure-additive coverings and measurable selectors, Dissertationes Math. 260 (1987).

H. Friedman, [1980] A consistent Fubini-Tonelli theorem for nonmeasurable functions, Illinois J. Math. 24 (1980), 390-395.

M. Gitik and S. Shelah, [1988] Forcing with ideals and simple forcing notions, preprint.

K. Gödel, [1964] What is Cantor's Continuum Problem?, Philosophy of Mathematics, edited by P. Benacerraf and H. Putnam, Prentice-Hall, Englewood Cliffs, N.J., 1964.

S. Gudder, [1984] Probability manifolds, J. Math. Phys. 25 (1984), 2397-2401.

T. Jech, [1978] Set theory (Academic Press, New York, 1978).

K. Kunen, [1984] Random and Cohen reals, Chapter 20 of Handbook of Set-Theoretic Topology, edited by K. Kunen and J. E. Vaughan, Elsevier Science Publishers, 1984.

I. Pitowsky, [1983] Deterministic models of spin and statistics, Phys. Rev. D 27 (1983), 2316-2326.

A. Shiryayev, [1984] Probability, Springer-Verlag, New York, 1984.

R. Solovay, [1970] A model of set theory in which every set of reals is Lebesgue measurable, Ann. of Math. 92 (1970), 1-56.

[1971] Real-valued measurable cardinals, Axiomatic Set Theory, edited by D. Scott, Proc. Sympos. Pure Math., vol. 13, part 1, Amer. Math. Soc., Providence, R.I., 1971, pp. 397428.

S. Ulam, [1930] Zur Masstheorie in der algemeinen Mengenlehre, Fund. Math. 16 (1930), 140-150.

Department of Mathematics, Brandeis University, Waltham, Massachusetts 02154

Current address: 67 Baxter Street, Melrose, Massachusetts 02176 